\title{
Повыиение точности расчета систем автоматического управления с запаздыванием
}

\author{
B.3. Тхан 1, аспиранm, dungvietthan@gmail.com \\ Ю.Н. Дементьев 1, к.т.н., доиент, dementev@tрu.ru \\ B.И. Гончаров 1, д.m.н., npoppeccop, gvi@tpu.ru
}

1 Наииональный исследовательский Томский политехнический университет, 634050, г. Томск, Россия

В статье рассматриваются вопросы точности задач синтеза систем автоматического управления объектами с запаздыванием. Они охватывают традиционный подход на основе аппроксимации передаточной функции звена запаздывания его дробно-рациональным выражением, а также оригинальный путь, позволяющий сохранить точное описание звена запаздывания при решении задач синтеза регуляторов систем автоматического управления.

В работе выбран последний вариант, который базируется на привлечении частного случая преобразования Лапласа, обеспечивающего переход к описанию динамических систем в форме функций-изображений, имеющих вещественную переменную. Этот численный метод дает возможность получать дискретные модели динамических систем, сочетая преимущества численных методов и цифровых вычислительных средств.

На основе численного метода рассмотрены три актуальные задачи синтеза и исследования систем указанного класса. Первая из них связана с аппроксимацией звена запаздывания. Представлен сравнительный анализ подходов, базирующихся на отрезках ряда Тейлора, дроби Паде и численного метода. Выявлены преимущества последнего: более высокая точность и, главное, возможность перераспределения погрешности приближения в области малых/больших значений времени. В рамках второй задачи показана возможность формирования и решения уравнения синтеза систем автоматического управления без аппроксимации звена запаздывания, обеспечивая достижение потенциально возможной точности синтеза. Третья задача раскрывает возможности численного метода в оценивании точности синтеза систем автоматического управления и сравнения нескольких вариантов.

Предложен вариант оценивания, не требующий аппроксимации звена запаздывания. Результаты проиллюстрированы примерами расчетов.

Ключевые слова: объект с запаздыванием, синтез регуляторов, аппроксимация звена запаздывания, преобразования Лапласа, повышение точности САУ с запаздыванием.

Системы автоматического управления (САУ) объектами с запаздыванием составляют обширный класс индустриальных систем управления, что отражает постоянный интерес к ним в течение десятилетий. Их особенность состоит в наличии звена запаздывания, описываемого передаточной функцией $e^{-p \tau}$, которая не позволяет использовать классические методы синтеза и исследования систем управления. Для снятия препятствий обычно используется прием аппроксимации функции $e^{-p \tau}$ подходящим дробно-рациональным выражением, что позволяет привлекать стандартные методы расчета САУ [1-6].

Несмотря на очевидность и простоту этого пути, у него имеются негативные стороны. Во-первых, в исходном уравнении синтеза САУ $W_{\text {ж }}^{3}(p) \simeq W_{\mathrm{c}}^{3}(p)$, в котором передаточная функция $W_{\text {ж}}^{3}(p)$ описывает желаемую, эталонную систему, a $W_{\mathrm{c}}^{3}(p)$ - синтезируемую САУ, имеющую вид

$W_{\mathrm{c}}^{3}(p) \simeq \frac{W_{p}(p) W_{\text {нч }}(p) e^{-p \tau}}{1+W_{p}(p) W_{\text {нч }}(p) e^{-p \tau} k_{\mathrm{oc}}}$,

в случае замены составляющей $e^{-p \tau}$ конечномерным представлением появляется составляющая погрешности, определенная переходом от точного представления звена запаздывания к приближен- ному. Во-вторых, на этапе оценивания точности синтеза системы обычно стремятся получить ее переходную характеристику, которая позволяет найти прямые показатели качества. Однако здесь также приходится аппроксимировать звено запаздывания, так как иначе задача, за исключением простейших случаев, оказывается неразрешимой. В-третьих, для некоторых систем необходимо определять временные характеристики САУ с повышенной точностью при малых или больших значениях времени, что требует соответствующего инструмента при аппроксимации звена запаздывания.

Выделенные вопросы являются важными в практическом и теоретическом отношениях и потому являются предметом рассмотрения в настоящей работе. В рамках аналитических исследований получить приемлемые ответы на эти вопросы в общем случае невозможно, поэтому используется численный метод - вещчественный интерполяциионный метод (ВИМ) [7, 8].

\section{Предварительные замечания}

Для пояснения некоторых особенностей метода ВИМ приведем краткие сведения о нем. Метод базируется на вещественном интегральном преобразовании: 


$$
F(\delta)=\int_{0}^{\infty} f(t) e^{-\delta . t} d t, \delta \in[C, \infty), C \geq 0,
$$

которое ставит в соответствие оригиналу $f(t)$ изображение $F(\delta)$ в виде функции вещественной переменной $\delta$. Формулу прямого преобразования (2) можно рассматривать как частный случай прямого преобразования Лапласа при замене комплексной переменной $p=\delta+j \omega$ на вещественную $\delta \geq 0$. Еще один шаг, направленный на расширение возможностей метода, - переход от непрерывных функций $F(\delta)$ к их дискретным аналогам $F\left(\delta_{i}\right), i=1,2, \ldots, \eta$, с целью применения средств вычислительной техники и численных методов. Для этих целей в ВИМ введены численные характеристики $\left\{F\left(\delta_{i}\right)\right\}_{\eta}$, которые получают как совокупность значений функции $F(\delta)$ в узлах $\delta_{i}, i=1,2, \ldots, \eta$.

Основные достоинства непрерывных моделей $F(\delta)$ и их дискретных представлений $F\left(\delta_{i}\right), i=1,2$, ..., $\eta$, связаны, во-первых, с тем, что они описывают динамическую систему в области изображений, обеспечивая простоту действий над такими моделями по сравнению с функциями времени. Вовторых, аргумент $\delta$ является вещественным, что позволяет привлекать хорошо разработанные численные методы функций вещественных переменных. Модели вида $F\left(\delta_{i}\right), i=1,2, \ldots, \eta$, распространяются как на передаточные функции $W(p)$, базирующиеся на преобразовании Лапласа, так и на изображения сигналов $F(p)=L\{f(t)\}$, приводя, когда это нужно, к моделям $W(\delta)$ и $F(\delta)$.

Перейдем к рассмотрению выделенных ранее вопросов, начиная с наиболее значимого - аппроксимации передаточной функции звена запаздывания.

\section{Аппроксимация звена запаздывания}

В настоящее время используют два основных метода аппроксимации звена запаздывания, отличающихся простотой вычислений: разложение передаточной функции $e^{-p \tau}$ в ряд Тейлора и представление этой функции в виде дроби Паде [9]. Оба метода в своей основе используют степенной ряд Тейлора, а приближения имеют вид

$$
\begin{aligned}
e^{-\tau p} & =\frac{1}{1+\tau p+\frac{1}{2}(\tau p)^{2}+\frac{1}{6}(\tau p)^{3}+\ldots}, \\
e^{-\tau p} & =\frac{1-\frac{1}{2} \tau p+\frac{1}{12}(\tau p)^{2}+\ldots}{1+\frac{1}{2} \tau p+\frac{1}{12}(\tau p)^{2}+\ldots} .
\end{aligned}
$$

Сравнивая представленные выражения, можно отметить, что аппроксимационные возможности второго варианта должны быть выше, так как он имеет большее число варьируемых коэффициентов. На основе ВИМ также можно использовать аппроксимации, имеющие преимущества по числу коэффициентов. Для наглядного сравнения возможностей трех выделенных методов воспользуемся расчетным примером.

Для простоты примем запаздывание равным $1 \mathrm{c}$. Тогда передаточная функция звена будет иметь вид $W(p)=e^{-p}$. В качестве аппроксимирующего выражения примем распространенную функцию второго порядка. Результаты приближения принятой формой для ряда Тейлора и дроби Паде представлены соответственно выражениями

$$
e^{-p} \simeq \frac{1}{0.5 p^{2}+p+1} \text { и } e^{-p} \simeq \frac{0.083 p^{2}-0.5 p+1}{0.083 p^{2}+0.5 p+1} \text {. }
$$

Соответствующие переходные характеристики показаны на рисунке 1 . Их сравнение позволяет выделить достоинства и недостатки этих вариантов. Отрезок ряда Тейлора имеет хорошую точность при малых значениях времени, однако он проигрывает аппроксимации Паде при больших значениях времени. У последнего имеется несомненное достоинство - повышенная точность при $t>\tau$, однако заметен и недостаток, связанный с ненулевым начальным значением, определенным принятой структурой.

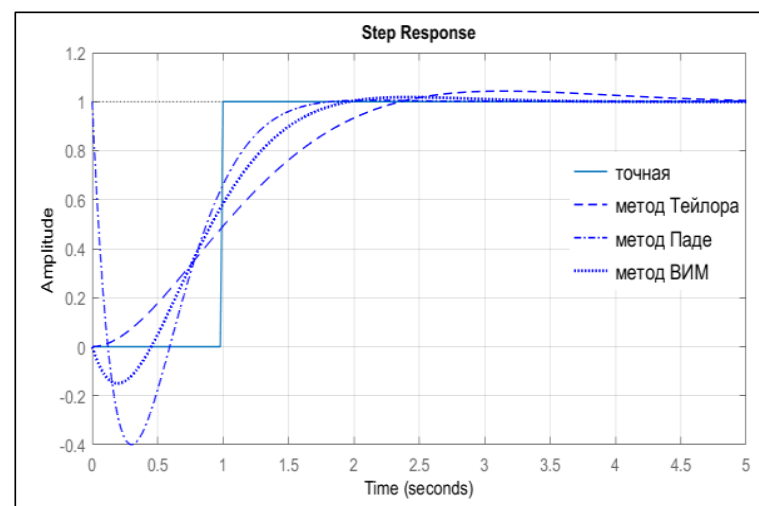

Рис. 1. Переходные характеристики звена запаздывания и аппроксимируюших выражений на основе ряда Тейлора, дроби Паде и ВИМ

Fig. 1. Transient characteristics of the time delay object and approximating expressions based on the Taylor series, Padé fraction and RIM

Перейдем к рассмотрению возможностей третьего метода - ВИМ. В качестве аппроксимирующей функции примем выражение

$$
W_{\text {пр. }}(p)=\frac{b_{1} p+b_{0}}{a_{2} p^{2}+a_{1} p+1},
$$

которое позволяет избежать ненулевого начального значения в области времени. Задача заключается в вычислении коэффициентов выбранной дроби. Представим ее решение достаточно подробно, настолько, чтобы была возможность проверки алгоритма и всех вычислений. Значение коэффициента $b_{0}$ найдется по установившемуся режиму: $b_{0}=1$. Для вычисления оставшихся трех коэффициентов воспользуемся численными расче- 
тами ВИМ. С этой целью заменим комплексную переменную $p=\delta+j \omega$ на вещественную $\delta \in[C, \infty]$, $C \geq 0$, и по вещественной функции $W(\delta)=e^{-\delta}$ найдем три узла интерполяции $\delta_{i}, i=1,2,3$, используя рекомендации из [7]. Получаем $\delta_{1}=0.1, \delta_{2}=0.2$, $\delta_{3}=0.3$, что позволяет найти численную характеристику $\left\{W\left(\delta_{i}\right)\right\}_{3}$, подставляя значения узлов в исходную функцию: $\left\{W\left(\delta_{i}\right)\right\}_{3}=\{0,9048 ; 0,8187$; $0,7408\}$. Это дает возможность сформировать систему уравнений вида $e^{-\delta_{i}}=\frac{b_{1} \delta_{i}+1}{a_{2} \delta_{i}^{2}+a_{1} \delta_{i}+1}, i=\overline{1,3}$, развернутая форма которой и ее решение приведены далее:

$$
\left\{\begin{array}{l}
0.9048=\frac{b_{1} \delta_{1}+1}{a_{2} \delta_{1}+a_{1} \delta_{1}+1} ; \\
0.8187=\frac{b_{1} \delta_{2}+1}{a_{2} \delta_{2}+a_{1} \delta_{2}+1} ; \Rightarrow\left\{\begin{array}{l}
b_{1}=-0.317 ; \\
a_{1}=0.184 ; \\
a_{2}=0.683 .
\end{array}\right. \\
0.7408=\frac{b_{1} \delta_{3}+1}{a_{2} \delta_{3}+a_{1} \delta_{3}+1} .
\end{array}\right.
$$

Приближенная передаточная функция звена запаздывания имеет вид $e^{-p} \simeq \frac{-0.317 p+1}{0.184 p^{2}+0.683 p+1}$, а ее переходная характеристика представлена на рисунке 1.

Из сопоставления временных характеристик можно сделать определенные выводы, позволяющие выбрать предпочтительный вариант аппроксимации при решении конкретных задач. Особенности двух первых методов в основном достаточно ясны, поэтому остановимся на возможностях ВИМ. Его привлекательность в том, что он позволяет перераспределять погрешность на участках малых и больших значений времени [10]. Условной границей между ними можно считать время, соответствующее величине запаздывания.

Для повышения точности в области малых или больших значений времени используется инструментальная переменная $\delta_{1}$, которая в обычных случаях определяет значения всех узлов интерполирования: $\delta_{i}=i \delta_{1}, i=1,2,3, \ldots$ [7].

Для демонстрации возможности коррекции точности по участкам приведем результаты приближения звена запаздывания прежней дробно-рациональной структурой с различными значениями переменной $\delta_{1}=0.05 ; 1.0 ; 1.5$ (см. рис. 2 ).

Из рисунка достаточно ясно видно, что в рамках ВИМ существует инструмент, который позволяет изменять погрешность приближения в области малых/больших значений времени, что важно, например, при исследовании систем повышенной точности. Механизм перераспределения погрешности по интересующему интервалу, например $[0 ; 2 \tau]$, следует непосредственно из соотношения (2), которое в обозначениях рассматриваемой задачи имеет вид

$$
W\left(\delta_{1}\right)=\delta_{1} \int_{0}^{\infty} h(t) e^{-\delta_{1} t} d t, \delta_{1}>0,
$$

где функция $h(t)$ - приближенная переходная характеристика. В этом интегральном соотношении функцию $e^{-\delta_{1} t}$ можно рассматривать в качестве весовой, которая позволяет изменять фильтрующие свойства. Например, при увеличении значения $\delta_{1}$ величина $W\left(\delta_{1}\right)$ будет определяться в основном начальной частью функции $h(t)$, что предопределяет снижение погрешности аппроксимации в области малых значений. Естественно, что погрешность при больших значениях времени будет возрастать вплоть до появления расходящегося процесса.

Справедливо и обратное - при уменьшении величины $\delta_{1}$ будет снижаться погрешность в области больших значений времени. Имеется и промежуточный вариант. Он ориентирован на повышение величины производной функции переходных характеристик в окрестности $t=\tau$, что в конечном итоге сказывается на точности приближения в указанной области. Но эта задача более сложная, так как требует неравномерного распределения узлов.

\section{Оценивание точности при решении задач синтеза}

Результаты синтеза САУ можно оценивать в комплексной, частотной и временной областях. Наиболее часто обращаются к функциям времени, обычно к переходным характеристикам, которые позволяют сравнивать по ним свойства эталонных и синтезированных систем, в том числе по их показателям качества. При этом задача сводится к обращению преобразования Лапласа $h_{\mathrm{c}}(p)=$ $=L^{-1}\left\{W_{\mathrm{c}}^{3}(p) / p\right\}$, которое является моделью синтезированной системы в форме изображения пере-

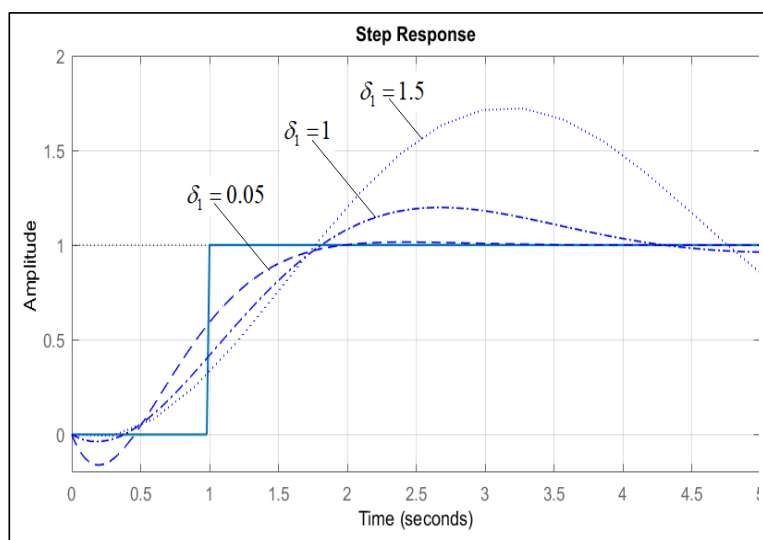

Рис. 2. Переходные характеристики аппроксимаций звена запаздывания по методу ВИМ при различных узлах интерполирования

Fig. 2. Transient characteristics of the time delay object approximations by the RIM method for various interpolation points 
ходной характеристики $h_{\mathrm{c}}(t)$. Однако этот путь в случае САУ с запаздыванием требует выполнения дополнительного этапа - замены выражения $e^{-\delta_{1} t}$ рациональной дробью, так как отсутствуют процедуры обратного преобразования изображений $W_{\mathrm{c}}^{3}(p) / p$, имеющих указанные особенности.

Сказанное свидетельствует о том, что при аппроксимационном пути получения оригинала $h_{\mathrm{c}}(t)$ по его изображению $W_{\mathrm{c}}^{3}(p) / p$ различие характеристик синтезированной $h_{\mathrm{c}}(t)$ и желаемой $h_{\text {ж }}(t)$ систем $\Delta h(t)=h_{ж}(t)-h_{\mathrm{c}}(t)$ будет содержать две составляющие: $\Delta h_{1}(t)+\Delta h_{2}(t)=\Delta h(t)$. Первая возникает из-за отсутствия точного решения уравнения синтеза. Вторая образуется как следствие этапа аппроксимации, что не позволяет в полной мере оценить точность решения синтеза по функции $\Delta h(t)$.

Для поиска путей уменьшения влияния составляющей $\Delta h_{2}(t)$ на общую величину $\Delta h(t)$ были проведены численные эксперименты, направленные на установление порядка аппроксимирующей функции $W_{\text {пр }}(p)=\frac{B_{m}(p)}{A_{n}(p)}$, при котором погрешность аппроксимации $\Delta h_{2}(t)$ будет значительно меньше интересующей нас ошибки $\Delta h_{1}(t)$. С этой целью для задачи синтеза САУ с желаемыми показателями перерегулирования и времени установления были получены переходные характеристики с различными вариантами дробей, аппроксимирующих звено запаздывания $e^{-3 p}$. Были исследованы дроби со следующими структурными параметрами: $m=0$, $n=1 ; m=1, n=2 ; m=2, n=3$. Для контроля точности сравнивались величины перерегулирования желаемой системы $\sigma_{ж}=5 \%$ и синтезированной $\sigma_{c}$, для чего использовалась оценка $\Delta \sigma=\sigma_{ж}-\sigma_{\mathrm{c}}$. Установлено, что уже при структуре $m=1, n=2$ достигнута приемлемо малая погрешность $\Delta h_{2}(t)$, так как оценка $\Delta \sigma$ оказалась равной $0,1 \%$. Этот результат свидетельствует о том, что для большинства практических задач аппроксимация звена запаздывания с параметрами $m=1, n=2$ позволяет оценивать точность синтеза САУ в области времени.

Рассмотренный вариант оценивания точности синтезированных САУ вполне приемлем для практики, однако следует иметь в виду, что он сохраняет в составе общей погрешности $\Delta h(t)$ составляющую $\Delta h_{2}(t)$, которая по сути является ошибкой вычислений. Для высокоточных систем могут потребоваться сопоставление и выбор одного из двух близких вариантов САУ. В этих случаях желательно полностью устранить влияние составляющей $\Delta h_{2}(t)$. Такой путь исследования точности может быть реализован на основе ВИМ. Рассмотрим эту возможность.

Одна из особенностей метода состоит в том, что он позволяет переходить к моделям вида $F(\delta)$ и $\left\{F\left(\delta_{i}\right)\right\}_{\eta}$, которые могут быть точными для передаточных функций, содержащих трансцендентные выражения, в том числе вида $e^{-p t}$ [7]. Это относится, в частности, к уравнению синтеза (1). Учитывая сказанное, положим, что задача синтеза решена, имеются вещественные передаточные функции желаемой $W_{ж}^{3}(\delta)$ и синтезированной системы $W_{\mathrm{c}}^{3}(\delta)$. Теперь можно найти погрешность синтеза в виде функции

$$
\Delta W^{3}(\delta)=W_{ж}^{3}(\delta)-W_{\mathrm{c}}^{3}(\delta) .
$$

График такой функции для конкретной задачи показан на рисунке 3. График демонстрирует области, в которых погрешность $\Delta W^{3}(\delta)$ мала либо, наоборот, велика. Эта особенность графического представления погрешности позволяет выделять области, имеющие большие отклонения, и намечать меры для их снижения за счет изменения значений узлов интерполирования. Напомним, что в ВИМ существует механизм, который позволяет изменять погрешность в области времени на начальном (или конечном) участке интервала $\left[0, t_{\mathrm{y}}\right]$ путем соответствующего смещения узлов $\delta_{i}, i=\overline{1, \eta}$.

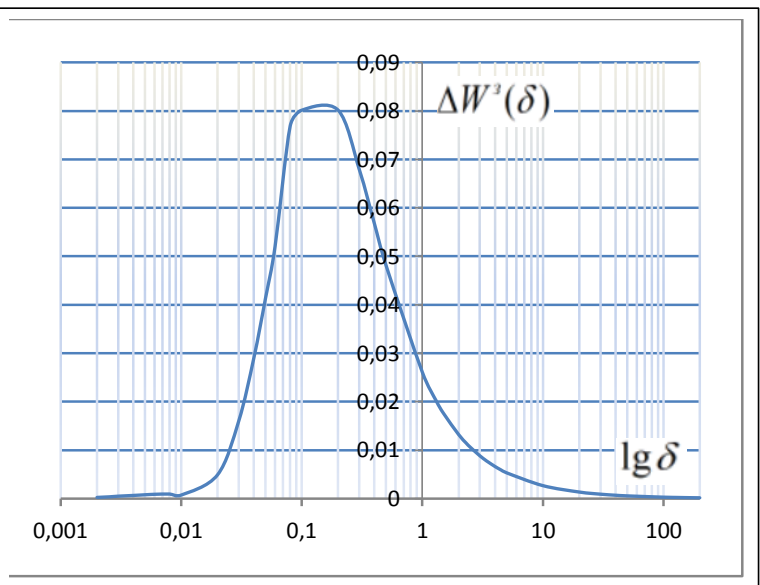

Рис. 3. График отклонения $\Delta W^{3}(\delta)$ синтезированной передаточной функции $W_{\mathrm{c}}^{3}(\delta)$ от желаемой $W_{ж}^{3}(\delta)$ замкнутой системы

Fig. 3. The graph of the deviation of the synthesized transfer function $W_{\mathrm{c}}^{3}(\delta)$ from the desired closed system $W_{\%}^{3}(\delta)$

Функция $\Delta W^{3}(\delta)$ может быть использована для получения численных оценок свойств САУ, например, в виде $\Delta W^{3}=\max _{\delta}\left|\Delta W^{3}(\delta)\right|$,

$$
\Delta W^{3}\left(\delta_{i}\right)=\int_{0}^{\infty} \Delta k^{3}(t) e^{-\delta_{i} t} d t, \delta_{i}>0 \text { и т.п. }
$$

Смысл таких оценок вытекает из взаимосвязи функций $\Delta W_{\mathrm{c}}^{3}(\delta)$ и $\Delta k^{3}(t)$, определенных соотношением

$$
\begin{aligned}
& \Delta W^{3}(\delta)=W_{\text {ж }}^{3}(\delta)-W_{\mathrm{c}}^{3}(\delta)= \\
& \left.=\int_{0}^{\infty} k_{\text {ж}}^{3}(t) e^{-\delta t} d t\right)-\int_{0}^{\infty} k_{\mathrm{c}}^{3}(t) e^{-\delta t} d t=\int_{0}^{\infty} \Delta k^{3}(t) e^{-\delta t} d t,
\end{aligned}
$$


в котором $k_{\text {ж }}^{3}(t)$ и $k_{\mathrm{c}}^{3}(t)$ - импульсные переходные характеристики.

И еще один вывод, который напрашивается при просмотре графика $\Delta W^{3}(\delta)$. Он связан с возможностью получения наилучшего равномерного приближения функции $W_{\mathrm{c}}^{3}(\delta)$ к $W_{\text {ж}}^{3}(\delta)$. Чебышев П.Л. доказал, что такие приближения достигаются, если, в частности, величины всех отклонений функции $\Delta W^{3}(\delta)$ (в обозначениях рассматриваемой задачи) равны между собой, а их знаки чередуются. Это явление, получившее название альтернанса Чебышева, в данном случае не соблюдается, что объясняется тем, что лучшее решение выбиралось по переходным характеристикам синтезированных САУ. Этот выбор можно формализовать, обеспечивая приближению к Чебышевскому альтернансу функции времени, например $\Delta k^{3}(t)$. Инструментом для этого может служить распределение узлов интерполирования [9]. Некоторые из изложенных сведений были использованы для формирования критерия робастности САУ с запаздыванием [11]. Он позволяет оценивать изменения параметров объекта управления, которые приводят к изменениям свойств САУ, в частности, перерегулирования.

\section{Заключение}

В работе рассмотрены вопросы оценивания точности синтеза систем управления с запаздыванием. Как результат можно выделить два основных вывода. Первый из них связан с аппроксимацией передаточной функции звена запаздывания. Он открывает новую возможность в аппроксимации звена запаздывания - перераспределение погрешности между участками малых и больших значений времени. Этот вывод важен еще и тем, что он распространяется на задачу исследования точности решения уравнения синтеза. Второй результат также связан с оценками точности синтезированных САУ. Он дает возможность сравнивать не- сколько вариантов синтеза по точным характеристикам без привлечения аппроксимации звена запаздывания.

Некоторые из изложенных сведений были использованы для формирования критерия робастности САУ с запаздыванием [8]. Он позволяет оценивать изменения параметров объекта управления, которые приводят к изменениям свойств САУ, в частности, перерегулирования.

Работа выполнена при поддержке профессора Нгуен Тхань Нги.

\section{Лuтература}

1. Денисенко В.В. Компьютерное управление технологическим процессом, экспериментом, оборудованием. М.: Горячая линия-Телеком, 2009. $608 \mathrm{c}$.

2. Громов Ю.Ю., Земской Н.А., Лагутин А.В., Иванова О.Г., Тютюнник В.М. Системы автоматического управления с запаздыванием. Тамбов: Изд-во Тамб. гос. техн. ун-та, 2007. $76 \mathrm{c}$.

3. Курганов В.В., Цавнин А.В. Управление объектом с запаздыванием // Автоматика и программная инженерия. 2015. № 2. C. $9-13$.

4. Лукас В.А. Теория автоматического управления. М.: Недра, 1990. 416 с.

5. Стопакевич А.А. Проектирование робастных регуляторов объектами с большим запаздыванием // Восточно-европейский журнал передовых технологий. 2016. Т. 1. № 2. С. 48-56.

6. Федосеев Б.Т. Об анализе САР со звеном задержки в контуре. URL: http://model.exponenta.ru/ (дата обращения: 28.03.2018).

7. Goncharov V.I., Aleksandrov I.A., Rudnicki V.A., Liepinsh A.V. Real Interpolation Method for Automatic Control Problem Solution. Saarbrucken, LAP LAMBERT Academic Publishing, 2014, $291 \mathrm{p}$.

8. Гончаров В.И. Вещественный интерполяционный метод синтеза систем автоматического управления. Томск: Изд-во ТПУ, 1995. $100 \mathrm{c}$.

9. Бейкер Дж., Грейвс-Моррис П. Аппроксимации Паде. М.: Мир, 1986. 502 c.

10. Goncharov V., Rudnicki V. Real interpolation method in automatic control systems self-adjustment problem // Systems Science, 2010, vol. 36, no. 3, pp. 35-37.

11. Белихмайер М.Я., Гончаров В.И. Синтез корректирующих устройств систем автоматического управления на основе равномерного приближения // Автоматика и телемеханика. 1997. № 5. C. 3-11.

\section{Improving the accuracy calculation of time delay automatic control}

V.D. Than ${ }^{1}$, Postgraduate Student, dungvietthan@gmail.com

Yu.N. Dementev ${ }^{1}$, Ph.D. (Engineering), Associate Professor, dementev@tpu.ru

V.I. Goncharov ${ }^{1}$, Dr.Sc. (Engineering), Professor, gvi@tpu.ru

${ }^{1}$ National Research Tomsk Polytechnic University, Tomsk, 634050, Russian Federation

Abstract. The article considers accuracy problems of synthesis automatic control systems (ACS) with time delay. The questions cover the traditional approach based on approximation of a delay element transfer function by a rational fractional 
expression, as well as the original way that allows keeping an accurate description of the delay element when solving the problems of ACS regulator synthesis.

The authors choose the last option, which is based on using a special case of the Laplace transform that provides a transition to the description of dynamical systems in the form of image functions with a real variable. This numerical method makes it possible to obtain discrete models of dynamic systems, combining the advantages of numerical methods and digital computing tools.

Based on a numerical method the authors consider three topical problems of synthesis and research of the indicated class systems. The first one is related to a delay element approximation. The paper gives a comparative analysis of the approaches based on segments of the Taylor series, Padé fractions and the numerical method. The advantages of the latter are: higher accuracy and, most importantly, it is possible to redistribute the approximation error in the region of small/large values of time.

Within the framework of the second problem the paper shows the possibility of forming and solving the synthesis equation for an automatic control system without approximation of a delay element ensuring the achievement of the potentially possible synthesis accuracy. The third problem reveals the possibilities of the numerical method in estimating the accuracy of automatic control system synthesis and the comparison of several options. The authors propose a variant of estimation that does not require the approximation of the delay element. A result is graphically illustrated and proved by numbers of examples.

Keywords: delay element, regulator synthesis, delay element approximation, Laplace transform, time delay ACS accuracy improvement.

Acknowledgements. The work has been supported by Professor Nguyễn Thanh Nghị.

\section{References}

1. Denisenko V.V. Computer Control of a Technical Process, an Experiment, Equipment. Moscow, Hotline-Telecom Publ., 2009, 608 p.

2. Gromov Yu.Yu., Zemsky N.A., Lagutin A.V., Ivanova O.G., Tyutyunnik V.M. Automatic Control Systems with Delay. Tambov, TSTU Publ., 2007, 76 p.

3. Kurganov V.V., Tsavnin A.V. Controlling an object with delay. Automation and Software Engineering. 2015, no. 2, pp. 9-13.

4. Lucas V.A. Theory of Automatic Control. Moscow, Nedra Publ., 1990, 416 p.

5. Stopakevich A.A. Designing robust regulators with objects with a long delay. East-European J. of Advanced Technologies. 2016, vol. 1, no. 2, pp. 48-56.

6. Fedoseev B.T. On the Analysis of ATS with a Link of Delay in the Circuit. Available at: http://model.exponenta.ru/ (accessed March 28, 2018).

7. Goncharov V.I., Aleksandrov I.A., Rudnicki V.A., Liepinsh A.V. Real Interpolation Method for Automatic Control Problem Solution. Saarbrucken, LAP LAMBERT Academic Publ., 2014, 291 p.

8. Goncharov V. Real Interpolation Method of Synthesis of Automatic Control Systems. Tomsk, TPU Publ., 1995,100 p.

9. Baker J., Graves-Morris P. Padé Approximations. Cambridge Univ. Press, 1996, 764 p. (Russ. ed.: Moscow, Mir Publ., 1986, 502 p.).

10. Goncharov V., Rudnicki V. Real interpolation method in automatic control systems self-adjustment problem. Systems Science. 2010, vol. 36, no. 3, pp. 35-37.

11. Belichmayer M.Ya., Goncharov V.I. Synthesis of correcting devices of automatic control systems based on uniform approximation. Automation and Remote Control. 1997, no. 5, pp. 3-11 (in Russ.)

\section{Примеры бибциографического описания статьи}

1. Тхан В.З., Дементьев Ю.Н., Гончаров В.И. Повышение точности расчета систем автоматического управцения с запаздыванием // Программные продукты и системы. 2018. Т. 31. № 3. С. 521-526. DOI: $10.15827 / 0236-235 X .123 .521-526$.

2. Than V.D., Dementev Yu.N., Goncharov V.I. Improving the accuracy calculation of time delay automatic control. Software \& Systems. 2018, vol. 31, no. 3, pp. 521-526 (in Russ.). DOI: 10.15827/0236235X.123.521-526. 